\title{
FATORES ASSOCIADOS À REPRESENTATIVIDADE DA ZONA DE TRANSFORMAÇÃO EM EXAMES CITOPATOLÓGICOS DO COLO UTERINO
}

\author{
Vanessa Aparecida Gasparin ${ }^{1}$, Érica de Brito Pitilin², Rafaela Bedin ${ }^{3}$, Fernanda Karla Metelski ${ }^{4}$, Daniela Savi \\ Geremia $^{5}$, Claudio Claudino da Silva Filho ${ }^{6}$
}

\begin{abstract}
RESUMO: Objetivou-se analisar os fatores associados à representatividade da Zona de Transformação em exames citopatológicos para controle do câncer do colo do útero.Trata-se de um estudo retrospectivo de caráter quantitativo realizado por meio das análises dos exames citopatológicos registrados no Sistema de Informação do Câncer em 2014. A amostra final resultou em 1.157 laudos. Para a análise dos dados foi utilizado o software Statistical Package for the Social Sciences. Do total de exames, 96,88\% foram realizados para o controle e rastreamento da doença e $24,3 \%$ não apresentaram a zona de transformação. Os fatores que influenciaram a representatividade da zona de transformação foram idade entre 25 a 64 anos, uso de contraceptivo oral, terapia de reposição hormonal e epitélio metaplásico. Tais fatores podem ser considerados facilitadores para a captação de uma amostra satisfatória com a possível diminuição de resultados falso-negativos, o que acarretaria o retardo do tratamento precoce do câncer do colo uterino.
\end{abstract}

DESCRITORES: Câncer do colo do útero; Programas de rastreamento; Esfregaço vaginal.

\section{FACTORS ASSOCIATED WITH THE REPRESENTATIVENESS OF THE TRANSFORMATION ZONE IN CYTOPATHOLOGICAL CERVICAL TESTS}

\begin{abstract}
The objective was to analyze the factors associated with the representativeness of the Transformation Zone in cervical screening tests. A retrospective and quantitative study was undertaken through the analyses of the cytopathology tests registered in the Cancer Information System in 2014. The final sample resulted in 1,157 reports. For the data analysis, the software Statistical Package for the Social Sciences was used. Of all tests, $96.88 \%$ were undertaken for disease control and screening and $24.3 \%$ did not present the transformation zone. The factors that influenced the representativeness of the transformation zone were age between 25 and 64 years, use of oral contraception, hormone replacement therapy and metaplastic epithelium. These factors can be considered facilitators to capture a satisfactory sample with the possible reduction of false-negative results, which would entail a delay for the early treatment of cervical cancer.

DESCRIPTORS: Uterine cervical neoplasms; Mass screening; Vaginal smears.
\end{abstract}

\section{FACTORES ASOCIADOS A LA REPRESENTATIVIDAD DE LA ZONA DE TRANSFORMACIÓN EN EXÁMENES DE CITOPATOLOGÍA DEL CUELLO UTERINO}

RESUMEN: Estudio cuya finalidad fue analizar los factores asociados a la representatividad de la Zona de Transformación en exámenes de citopatología para control del cáncer de cuello del útero. Es un estudio retrospectivo de carácter cuantitativo realizado por medio de los análisis de exámenes de citopatología registrados en el Sistema de Información del Cáncer en 2014. La muestra final resultó en 1.157 informes. Para el análisis de los datos, se utilizó el software Statistical Package for the Social Sciences. Del total de exámenes, $96,88 \%$ fueron realizados para el control y rastreo de la enfermedad y $24,3 \%$ no presentaron la zona de transformación. Los factores que influenciaron la representatividad de la zona de transformación fueron edad entre 25 y 64 años, uso de anticonceptivo oral, terapia de reposición hormonal y epitelio metaplásico. Se puede considerar esos factores como facilitadores para la captación de una muestra satisfactoria con la posible disminuición de resultados falso-negativos, lo que resultaría el retraso del tratamiento precoz del cáncer de cuello uterino.

DESCRIPTORES: Cáncer de cuello de útero; Programas de rastreo; Examen vaginal.

${ }^{1}$ Enfermeira. Mestranda em Enfermagem. Universidade Federal do Rio Grande do Sul. Porto Alegre, RS, Brasil.

${ }^{2}$ Enfermeira. Doutoranda em Enfermagem. Docente da Universidade Federal da Fronteira Sul. Chapecó, SC, Brasil.

${ }^{3}$ Enfermeira. Pós-graduanda. Instituto Fisiomar. Chapecó, SC, Brasil.

${ }^{4}$ Enfermeira. Mestre em Políticas Sociais e Dinâmicas Regionais. Docente da Universidade do Estado de Santa Catarina. Chapecó, SC, Brasil.

${ }^{5}$ Enfermeira. Doutora em Saúde Coletiva. Docente da Universidade Federal da Fronteira Sul. Chapecó, SC, Brasil.

${ }^{6}$ Enfermeiro. Doutorando em Enfermagem. Docente da Universidade Federal da Fronteira Sul. Chapecó, SC, Brasil.

Autor Correspondente:

Vanessa Aparecida Gasparin

Universidade Federal da Fronteira Sul

R. Clevelandia, 102D - 89801-560 - Chapecó, SC, Brasil

E-mail: vaneapgasparin@gmail.com.
Recebido: 17/12/2015

Finalizado: $25 / 06 / 2016$ 


\section{- INTRODUÇÃO}

O exame citopatológico do colo do útero configura-se como estratégia de rastreamento para a detecção precoce desse tipo de câncer. Conhecido também como Papanicolau, Pap Test ou Preventivo, tem como objetivo identificar lesões precursoras ou sugestivas de câncer a partir de células epiteliais há mais de 50 anos, sendo a primeira opção de escolha existente no mundo atualmente para a prevenção secundária do câncer ${ }^{(1,2)}$. Estudos comparativos temporais têm demonstrado uma redução significativa nas taxas de morbimortalidade após a introdução desse tipo de programa ${ }^{(3)}$. No Brasil, recomenda-se que esse exame seja realizado prioritariamente em mulheres entre 25 a 64 anos, com repetição a cada três anos após dois resultados normais consecutivos realizados no intervalo de um ano ${ }^{(4)}$.

Para tanto, uma adequada coleta do material é de suma importância para o êxito do diagnóstico. Quando correlacionado a uma cobertura populacional de $80 \%$ de adequabilidade amostral, reduz em até $90 \%$ as taxas de incidência do câncer do colo do útero ${ }^{(2)}$. Os fatores limitantes do efetivo potencial de rastreamento da citologia oncótica caracterizam-se pela amostra celular insuficiente e a preparação inadequada dos esfregaços, estando intimamente relacionados com a forma da coleta realizada pelos profissionais ${ }^{(5)}$. Assim, o profissional de saúde deve assegurar a garantia de um esfregaço satisfatório com a presença de células escamosas e glandulares em quantidade representativa, bem distribuídas, fixadas e com o alcance da Zona de Transformação (ZT), região onde localizam-se mais de $90 \%$ das lesões que antecedem o câncer de colo do útero ${ }^{(4)}$.

A ZT caracteriza-se pela intersecção do epitélio estratificado da ectocérvice com o epitélio colunar da endocérvice e sua presença na coleta dos exames tem sido considerada um indicador de qualidade de amostras satisfatórias ${ }^{(6)}$. A coleta insatisfatória representa gastos para o sistema de saúde, uma vez que não há restrição quanto ao pagamento desses exames. Ainda, caracteriza-se pelo desgaste físico da mulher, que ao retornar à unidade de saúde, deverá ser submetida a uma nova $\operatorname{coleta}^{(7)}$.

A ausência de ZT na amostra não é utilizada para classificá-la como insatisfatória, entretanto pode demonstrar a necessidade de monitoramento constante e qualificação dos profissionais ao realizarem a coleta do exame preventivo ${ }^{(4)}$. Alguns estudos vigentes na literatura analisaram os laudos citopatológicos traçando o perfil dos microrganismos encontrados e o grau de inflamação prevalentes nas células cervicais ${ }^{(3,8)}$. No entanto, são poucos os estudos publicados em relação ao tema até o momento que avaliam a qualidade da amostra do exame por meio da análise da representatividade da zona de transformação( ${ }^{(9)}$.

Mesmo com o aumento da cobertura do exame disponível nas unidades de saúde e da facilidade no acesso a esses serviços, o câncer do colo do útero configura-se na terceira maior causa de câncer nas mulheres e a quarta causa de morte entre elas, com estimativas de 17.540 novos casos por ano e um risco estimado de 17 casos a cada 100 mil mulheres $^{(4)}$. Logo, tal situação propõe a ocorrência de duas hipóteses: baixa qualidade na realização da coleta do exame ou falhas no seguimento das mulheres nos casos com lesões precursoras ${ }^{(5)}$.

Considerando que a realização da coleta do material citológico é majoritariamente realizada pelo enfermeiro, faz-se necessário avaliar a qualidade da assistência ofertada a partir da análise dos resultados dos exames e os fatores associados à ausência de células epiteliais coletadas, a fim de contribuir para que esses profissionais insiram novas condutas e rotinas no atendimento à mulher, podendo incrementar a capacidade de resolução da atenção primária à saúde ao identificar fatores passíveis de melhorias. Visando fortalecer e qualificar as ações no atendimento prestado às mulheres na prevenção e diagnóstico precoce do câncer de colo do útero, justifica-se a realização desta pesquisa.

Assim, esse estudo parte do pressuposto que os índices elevados de lesões precursoras do câncer de colo do útero podem ter sua origem nos determinantes relacionados aos resultados falso-negativos com falha na captura da região da zona de transformação. Diante do exposto, objetivou-se analisar os fatores associados à representatividade da ZT em exames citopatológicos para controle do câncer do colo do útero em um município polo do oeste catarinense. 
Trata-se de um estudo descritivo, retrospectivo de caráter quantitativo realizado a partir da análise dos laudos citopatológicos registrados no Sistema de Informação do Câncer (SISCAN) do Município de Chapecó-SC. Localizado na região oeste do estado, conta com 189.052 habitantes, sendo 95.700 mulheres ${ }^{(10)}$. Além disso, possui 29 Centros de Saúde da Família referência para a assistência à saúde ginecológica da mulher.

Para a determinação do cálculo amostral foi considerada a produção total de exames realizados no ano anterior à coleta de dados cadastrados no SISCAN do município equivalente a 19.296 exames. Para garantir maior confiabilidade estatística, foi acrescentando 3\% de erro de estimativa e confiabilidade de $97 \%$ para a definição da amostragem. Mediante a estratificação proporcional e representativa da produção de exames de cada unidade de saúde, foi definido o número de exames que foram analisados em cada uma. Assim, após o cálculo amostral, o número de laudos acessados no município totalizou 1.157 laudos.

A coleta de dados ocorreu no mês de agosto de 2015 e a seleção dos laudos deu-se pela sequência ordinal em que estes apareceram no sistema, iniciando-se pelo primeiro, seguido do segundo e assim sucessivamente, até atingir o número total apontado em cada unidade conforme o cálculo amostral. Dessa forma, os critérios de inclusão foram todos os laudos avaliados registrados no SISCAN e liberados para as pacientes no ano de 2014. Foi assegurado o anonimato de cada laudo sendo identificado por um código caracterizado pela inicial "L" da palavra "Laudo" acompanhado do algarismo arábico referente à ordem da coleta (L1, L2, L3...).

As variáveis estudadas foram aquelas que estavam descritas no sistema sendo idade, gestação confirmada, uso do Dispositivo Intrauterino (DIU), uso do contraceptivo oral, sangramento após relação sexual ou após menopausa, terapia de reposição hormonal, sinais de Doença Sexualmente Transmissível (DST), motivo da realização do exame, realização do exame anteriormente, inspeção do colo, avaliação da amostra, epitélios representativos, presença da ZT, diagnóstico descritivo, microbiologia e alterações celulares citológicas. Vale ressaltar que foram considerados como achados citológicos os resultados dos laudos que demonstravam citólise, hipotrofia, disceratose, macronucleose, escamas córneas, macrocitose, binucleação e halos perinucleares, por serem específicos para a patologia clínica.

Para a análise dos dados foi utilizado o software Statistical Package for the Social Sciences, versão 20.0. Para estudar a associação entre as variáveis independentes e a ocorrência ou não desfecho, foi realizado análise bivariada das variáveis em cada nível de determinação utilizando os testes Quiquadrado de Pearson, quando as variáveis apresentavam distribuição normal e Exato de Fisher para os dados não paramétricos.

A intensidade da associação foi avaliada por meio de estimativas de Odds Ratio. Para controlar o efeito de variáveis potencialmente confundidoras, realizou-se a análise multivariada (regressão logística múltipla). Para todos os testes estatísticos inferenciais foi utilizado nível de significância pré-estabelecido em $\mathrm{p}<0.05$. A qualidade do ajuste foi avaliada pelo teste de Hosmer-Lemeshow. O desenvolvimento desta pesquisa seguiu todas as normas com aprovação em 05/08/2015 pelo Comitê de Ética em Pesquisa com Seres Humanos da Universidade Federal da Fronteira Sul (UFFS), sob o Parecer nº 059401/2015 e Certificado de Apresentação para Apreciação Ética (CAAE) 46421815.0.0000.5564.

\section{- RESULTADOS}

Foram avaliados 1.157 laudos de exames citopatológicos do colo do útero. Destes, $70,1 \%(n=810)$ foram realizados em mulheres entre 25 a 64 anos, faixa etária considerada alvo das ações em saúde vigentes no país. Além disso, $22 \%(n=254)$ dos exames foram realizados em mulheres entre 15 e 24 anos e $8 \%$ acima de 65 anos (Figura 1). A média de idade foi de 37,5 (+/- 15,4 anos).

As principais características das mulheres segundo as variáveis estudadas a partir dos laudos dos exames citopatológicos estão na Tabela 1. Foi possível identificar que $96,7 \%(n=1.121)$ dos exames foram realizados para o rastreamento da doença e que $11,6 \%(n=134)$ das mulheres nunca haviam 


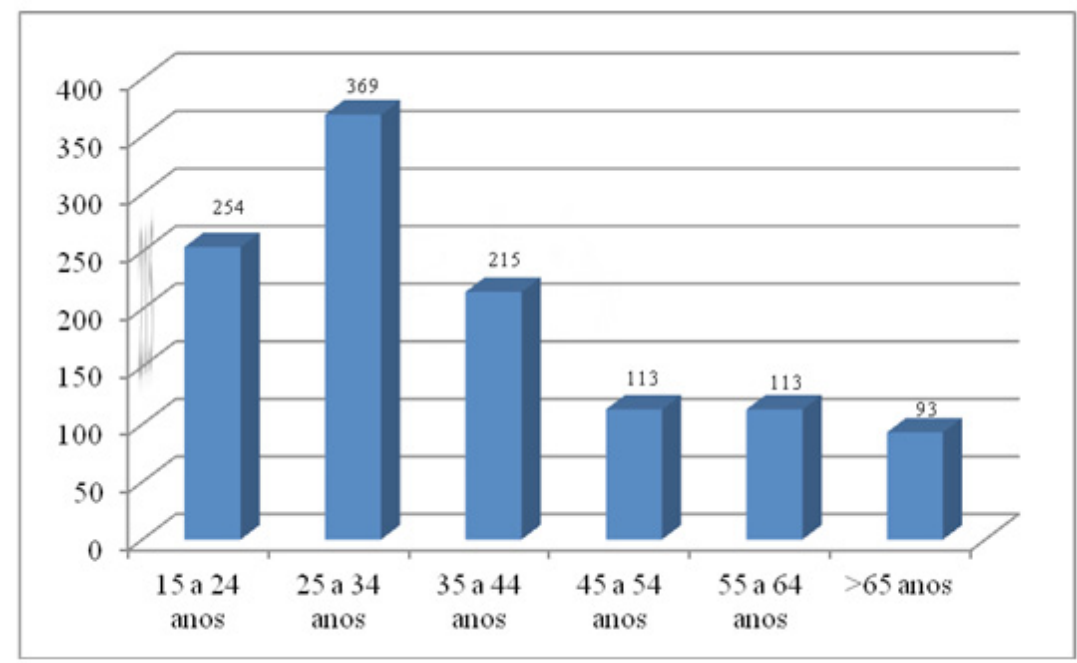

Figura 1 - Distribuição dos exames citopatológicos para o controle do câncer do colo do útero segundo grupo etário. Chapecó, 2015

realizado o exame antes.

Quanto aos resultados dos exames, 99\% da amostra selecionada foram satisfatórias e 75,6\% apresentaram o epitélio escamoso e glandular, conferindo ausência da ZT em $24,3 \%$ dos exames. Quanto ao diagnóstico, 41,4\% apresentavam dentro do limite da normalidade, seguido da atrofia com inflamação $(27,1 \%)$ e achados citológicos (11,7\%). A microbiota prevalente foram os Lactobacillussp (60,3\%) e o agente infeccioso a Gardnerellavaginalis (16,9\%) (Tabela 2).

Encontra-se na Tabela 3 a associação entre as variáveis estudadas e a representatividade da zona de transformação. Nessa primeira análise, as variáveis que mostraram associação foram: idade entre 25 a 64 anos, uso do contraceptivo oral, diagnóstico confirmado de gravidez, Terapia de Reposição Hormonal (TRH) e epitélio metaplásico.

Na Tabela 4 encontra-se o resultado da análise de regressão logística múltipla para controle dos efeitosdevariáveis potencialmenteconfundidoras. As variáveis que se mostraram estatisticamente associadas com a representatividade da ZT nesse modelo final foram: idade entre 25 a 64 anos, uso do contraceptivo oral, TRH e presença de epitélio metaplásico.
Tabela 1 - Características das mulheres segundo variáveis estudadas a partir dos exames citopatológicos para controle do câncer do colo do útero. Chapecó, SC, Brasil, 2015

\begin{tabular}{|c|c|c|}
\hline \multirow[t]{2}{*}{ Variáveis } & \multicolumn{2}{|c|}{ n= 1157 laudos } \\
\hline & Freq. & $\%$ \\
\hline \multicolumn{3}{|l|}{ Motivo exame } \\
\hline Rastreamento & 1121 & 96,7 \\
\hline Seguimento & 22 & 1,9 \\
\hline Repetição & 14 & 1,2 \\
\hline \multicolumn{3}{|c|}{ Já realizou o exame } \\
\hline Sim & 1023 & 88,4 \\
\hline Não & 134 & 11,6 \\
\hline \multicolumn{3}{|l|}{ Uso DIU* } \\
\hline Sim & 12 & 1 \\
\hline Não & 1145 & 99 \\
\hline \multicolumn{3}{|l|}{ Uso pílula } \\
\hline Sim & 549 & 47,5 \\
\hline Não & 608 & 52,5 \\
\hline \multicolumn{3}{|c|}{ Diagnóstico gravidez } \\
\hline Sim & 11 & 1 \\
\hline Não & 1146 & 99 \\
\hline \multicolumn{3}{|c|}{ Tratamento Radioterapia } \\
\hline Sim & 1 & 0,1 \\
\hline Não & 1156 & 99,9 \\
\hline \multicolumn{3}{|c|}{ Terapia Reposição Hormonal } \\
\hline Sim & 22 & 1,9 \\
\hline Não & 1135 & 98,1 \\
\hline \multicolumn{3}{|c|}{ Sangramento após relação sexual } \\
\hline Sim & 35 & 3 \\
\hline Não & 1122 & 97 \\
\hline \multicolumn{3}{|c|}{ Sangramento após menopausa } \\
\hline Sim & 8 & 0,7 \\
\hline Não & 1149 & 99,3 \\
\hline
\end{tabular}


Tabela 2 - Aspectos dos laudos citopatológicos de controle do câncer do colo do útero. Chapecó, SC, Brasil, 2015

\begin{tabular}{|c|c|c|}
\hline \multirow[t]{2}{*}{ Variáveis } & \multicolumn{2}{|c|}{$\mathrm{n}=1157$ laudos } \\
\hline & Freq. & $\%$ \\
\hline \multicolumn{3}{|l|}{ Aspecto do colo } \\
\hline Ausente & 30 & 2,6 \\
\hline Normal & 1042 & 90,1 \\
\hline Alterado & 80 & 6,9 \\
\hline Não visualizado & 5 & 0,4 \\
\hline \multicolumn{3}{|l|}{ Sugestivo DST* } \\
\hline Sim & 30 & 2,6 \\
\hline Não & 1127 & 97,4 \\
\hline \multicolumn{3}{|l|}{ Avaliação da amostra } \\
\hline Rejeitada & 3 & 0,3 \\
\hline Satisfatória & 1146 & 99 \\
\hline Insatisfatória & 8 & 0,7 \\
\hline \multicolumn{3}{|l|}{ Epitélio da amostra } \\
\hline Escamoso & 1146 & 99 \\
\hline Glandular & 866 & 75,6 \\
\hline Metaplásico & 203 & 17,7 \\
\hline \multicolumn{3}{|l|}{ Zona Transformação } \\
\hline Presente & 865 & 74,8 \\
\hline Ausente & 281 & 24,3 \\
\hline \multicolumn{3}{|l|}{ Diagnóstico } \\
\hline Dentro do limite da normalidade & 479 & 41,4 \\
\hline Metaplasia escamosa imatura & 67 & 5,8 \\
\hline Achados citológicos** & 135 & 11,7 \\
\hline Atrofia com inflamação & 313 & 27,1 \\
\hline Lesão Intraepitelial de baixo grau & 12 & 1 \\
\hline $\begin{array}{l}\text { Células atípicas de significado } \\
\text { indeterminado }\end{array}$ & 20 & 1,7 \\
\hline Lesão Intraepitelial de alto grau & 3 & 0,3 \\
\hline$>2$ associados & 117 & 10,2 \\
\hline \multicolumn{3}{|l|}{ Microbiota } \\
\hline Lactobacillussp & 698 & 60,3 \\
\hline $\operatorname{Cocos}$ & 2 & 0,2 \\
\hline Chlamydiasp & 1 & 0,1 \\
\hline Gardnerellavaginalis & 195 & 16,9 \\
\hline Candidaalbicans & 44 & 3,8 \\
\hline Bacilos & 145 & 12,5 \\
\hline Trichomonasvaginalis & 2 & 0,2 \\
\hline$>2$ associados & 105 & 9,1 \\
\hline
\end{tabular}

* Doença Sexualmente Transmissível

** citólise, hipotrofia, disceratose, macronucleose, escamas córneas, macrocitose, binucleação e halos perinucleares.
Tabela 3 - Análise bivariada da associação entre as variáveis estudadas a partir dos exames citopatológicos para o controle do câncer do colo do útero e a representatividade da zona de transformação encontrada no epitélio da amostra. Chapecó, SC, Brasil, 2015

\begin{tabular}{lccccc} 
Variáveis & \multicolumn{5}{c}{ Zona de Transformação (ZT) } \\
\cline { 2 - 6 } & Sim (n=865) & Não $(\mathbf{n = 2 8 1 )}$ & $\mathbf{p}$-Valor \\
\cline { 2 - 6 } & $\mathbf{n}$ & $\mathbf{\%}$ & $\mathbf{n}$ & $\mathbf{\%}$ & \\
\hline Público alvo & & & & & $\mathbf{0 , 0 1}$ \\
\hline Sim & 620 & 71,7 & 183 & 65,1 & \\
\hline Não & 245 & 28,3 & 98 & 34,9 & \\
\hline Uso DIU* & & & & & 0,769 \\
\hline Sim & 2 & 0,7 & 10 & 1,2 & \\
\hline Não & 855 & 98,8 & 279 & 99,3 & \\
\hline
\end{tabular}

\begin{tabular}{|c|c|c|c|c|}
\hline \multicolumn{5}{|c|}{ Uso Contr } \\
\hline Sim & 476 & 55 & 71 & 25,3 \\
\hline Não & 389 & 75 & 210 & 74,7 \\
\hline
\end{tabular}

Tratamento Radioterapia

\begin{tabular}{lcccc}
\hline Sim & 0 & 0 & 1 & 0,4 \\
\hline Não & 865 & 100 & 280 & 99,6
\end{tabular}

\begin{tabular}{|c|c|c|c|c|}
\hline \multicolumn{5}{|c|}{ Diagnóstico Gravidez } \\
\hline Sim & 5 & 0,6 & 5 & 1,8 \\
\hline Não & 860 & 99,4 & 276 & 98,2 \\
\hline
\end{tabular}

Sangramento após relação

0,481 sexual

\begin{tabular}{ccccc}
\hline Sim & 9 & 3,2 & 25 & 2,9 \\
\hline Não & 840 & 97,1 & 272 & 96,8
\end{tabular}

\begin{tabular}{lccccc}
\hline \multicolumn{2}{l}{ Sangramento após menopausa } & & 0,32 \\
\hline Sim & 0 & 0 & 7 & 2,5 & \\
\hline Não & 865 & 100 & 274 & 97,5 & \\
\hline TRH ${ }^{* *}$ & & & & & $\mathbf{0 , 0 0 0}^{* * * *}$ \\
\hline Sim & 12 & 1,4 & 8 & 2,8 & \\
\hline Não & 853 & 98,6 & 273 & 97,2 & \\
\hline
\end{tabular}

$\begin{array}{ll}\text { Aspecto do colo uterino } & 0,87\end{array}$

\begin{tabular}{ccccc} 
Normal & 799 & 92,8 & 235 & 93,3 \\
\hline Alterado & 62 & 7,2 & 17 & 6,7
\end{tabular}

$\begin{array}{lllll}\text { Alterado } & 62 & 7,2 & 17 & 6,7\end{array}$

Sinais sugestivos de DST*** $\quad 0,727$

\begin{tabular}{lccccc}
\hline Sim & 24 & 2,8 & 6 & 2,1 & \\
\hline Não & 841 & 97,2 & 275 & 97,9 & \\
\hline Epitélio metaplásico & & & & $\mathbf{0 , 0 0 0}^{* * * *}$ \\
\hline Sim & 202 & 23,4 & 1 & 0,4 & \\
\hline Não & 661 & 76,6 & 280 & 99,6 & \\
\hline Diagnóstico & & & & & 0,503 \\
\hline $\begin{array}{l}\text { Dentro da } \\
\text { normalidade }\end{array}$ & 362 & 41,8 & 117 & 41,6 & \\
\hline $\begin{array}{l}\text { Casos } \\
\text { positivos }\end{array}$ & 503 & 58,2 & 164 & 58,4 & \\
* Dispositivo Intra Uterino \\
** Terapia de Reposição Hormonal \\
*** Doença Sexualmente Transmissível \\
**** diferença significativa entre o grupo etário da amostra para \\
p<0,05 (teste exato de Fisher com correção de Yates).
\end{tabular}


Tabela 4 - Regressão logística dos fatores associados a representatividade da Zona de Transformação nas células epiteliais dos laudos citopatológicos. Chapecó, SC, Brasil, 2015

\begin{tabular}{lccc} 
Variável & OR Ajustado & IC 95\% & p-valor \\
\hline Uso do contraceptivo oral & 1,2 & $(1,37-2,98)$ & 0 \\
\hline Terapia de reposição hormonal & 0,4 & $(0,15-1,02)$ & 0,027 \\
\hline Epitélio metaplásico & 1,3 & $(0,19-2,96)$ & 0 \\
\hline Idade entre 25 a 64 anos & 0,6 & $(0,51-1,93)$ & 0,025
\end{tabular}

\section{- DISCUSSÃO}

Apesar da ampla cobertura de mulheres assistidas no âmbito da atenção primária e da facilidade do acesso ao exame, o percentual de mulheres que realizaram o preventivo neste estudo foi de $70,1 \%$, aquém das diretrizes propostas de $80 \%$ para impactar o perfil epidemiológico do câncer do colo do útero no país ${ }^{(11)}$. Outros estudos também evidenciaram baixa proporção de cobertura de exames em mulheres entre 25 a 64 anos $^{(11,12)}$.

A realização do exame em mulheres com menos 25 anos implicaria em um aumento significativo de diagnósticos de lesões de baixo grau que não são precursoras do câncer e irão retroceder espontaneamente na maioria dos casos, resultando em colposcopias e procedimentos diagnósticos e terapêuticos desnecessários ${ }^{(13)}$. Há vários fatos indicando que, direta ou indiretamente, o rastreamento em mulheres com menos de 25 anos não tem impacto na redução da incidência e/ou mortalidade por câncer do colo do útero, uma vez que 1,1\% dos casos de lesão invasora ocorrem nessas mulheres ${ }^{(14)}$. Por sua vez, não existe evidências que o rastreamento seja útil naquelas acima de 64 anos ${ }^{(15)}$.

Houveram mulheres deste estudo nunca haviam realizado o exame, à semelhança de outros estudos $^{(16,17)}$. Dentro os fatores associados à não adesão dessa prática encontram-se: medo, vergonha, desconforto físico, ausência de sintomas ou queixas e falta de informações ${ }^{(18)}$.

Ainda no que tange as características da população estudada, apenas $1 \%$ das mulheres que realizaram os exames neste estudo estavam no período gravídico, à luz da semelhança de outros estudos ${ }^{(19,20)}$. A situação gravídica não impede a mulher de desenvolver a neoplasia, nem de realizar o exame preventivo e tampouco interfere no decorrer favorável da mesma, tanto que a rotina do rastreamento de lesões precursoras do câncer do colo do útero em gestantes é a mesma realizada em mulheres no estado não gravídico $^{(4)}$. No município em estudo, as gestantes encontravam-se com seus exames citopatológicos realizados dentro da periodicidade preconizada pelo Ministério da Saúde (MS) ou os profissionais responsáveis pela coleta do exame não estavam realizando durante esse período.

Nesse estudo, 41,4\% das amostras apresentaram diagnóstico dentro do limite da normalidade, achado semelhante ao encontrado nos estudos realizados no Mato Grosso do Sul e São Paulo ${ }^{(21,22)}$. Por sua vez, as alterações celulares benignas foram as mais frequentes com destaque para as inflamações e os achados citopatológicos, assim como em outros estudos ${ }^{(8,23)}$. Pode-se inferir que a saúde reprodutiva das mulheres em questão está além das expectativas esperadas, uma vez que menos de $1 \%$ da população que realizou o exame apresentou complicações como sangramento após menopausa, tratamento com radioterapia, atipias em células escamosas seja de baixo ou alto grau, a julgar também pela baixa proporção de sinais sugestivos de DST e de colos visivelmente alterados.

Vale ressaltar que os achados citológicos encontrados nos exames deste estudo como citólise, disceratose, macrocitose, macronucleação, binucleação, entre outros são alterações celulares morfológicas que facilitam a Infecção pelo Papiloma Vírus (HPV) ${ }^{(24)}$. Os achados microbiológicos e infecciosos deste estudo como a presença de Lactobacillussp, e Gardnerella vaginalis, respectivamente, também foram encontrados nos estudos realizados na Paraíba e em Sergipe, classificados como achados normais por estarem inseridos na flora vaginal feminina e configurarem alterações no $\mathrm{pH}$ vaginal $^{(23,25)}$.

Ao avaliar os epitélios presentes na amostra deste estudo, foi possível identificar que $24,3 \%$ deles 
não apresentaram o epitélio glandular, reafirmando que a inexistência desse epitélio também revela a inexistência da ZT. A ausência dessa zona limita a visualização e a interpretação da amostra coletada o que contribui para um elevado índice de resultados falso-negativos estando associado à uma má qualidade da coleta dessas células ${ }^{(26)}$. Destaca-se que $62 \%$ das causas relacionadas aos resultados falso-negativos referem-se ao erro na coleta do material citológico ${ }^{(27)}$. Vale ressaltar que, neste estudo, menos de $1 \%$ das amostras foram rejeitadas ou insatisfatórias, caracterizando uma potencialidade da coleta realizada pelos enfermeiros.

Entre as variáveis que se mostraram estatisticamente associadas com o desfecho do estudo, o uso do contraceptivo oral apresentou 1,2 vezes mais chance de apresentar células representativas da ZT nas amostras dos exames. Acredita-se que haja uma influência da ação hormonal do estrogênio na distribuição das células basais e subcolunares do epitélio do colo uterino exteriorizando a localização dessa zona para uma região de fácil captura durante a coleta ${ }^{(7)}$. Assim como a ação hormonal do uso do contraceptivo oral, os hormônios decorrentes da TRH também apresentaram associação com a presença de células da ZT, corroborando que a ação do hormônio facilita a exteriorização dessas células facilitando a sua captura durante a coleta ${ }^{(4)}$. Tais achados não puderam ser comparados com outras pesquisas em virtude da escassez de estudos que utilizaram essa abordagem.

Outro aspecto evidenciado foi a faixa etária das mulheres compreendida entre 25 a 64 anos que apresentaram maior chance de ter a ZT facilmente capturada no momento da coleta do exame em relação aos demais grupos etários. Resultado semelhante foi encontrado em um estudo que, após a análise de 24.316 laudos, identificou uma diminuição no número de coletas de células da ZT conforme o aumento da faixa etária da população alvo ${ }^{(7)}$.

A presença do epitélio metaplásico também resultou na associação com a representatividade da ZT demonstrando a probabilidade de 1,3 vezes de ocorrer. A metaplasia escamosa consiste em um processo de transformação do epitélio glandular para epitélio escamoso que ocorre predominantemente em mulheres mais jovens, tornando-as vulneráveis ao desenvolvimento de lesões intraepiteliais cervicais e ao risco de infecção pelo $\mathrm{HPV}^{(28)}$. Além disso, a representatividade do epitélio metaplásico pode ser equivalente à presença de ZT, podendo ser utilizada também como parâmetro de qualidade da coleta do exame ${ }^{(26)}$. No entanto, alguns estudos apontam a baixa proporção de laudos com a captura desse epitélio metaplásico nas amostras coletadas podendo resultar em laudos falso-negativos que retardam o tratamento direcionado às mulheres com câncer de colo do útero ${ }^{(25,29)}$.

Considerando que $24,3 \%$ dos exames não apresentaram células representativas da ZT e que as diretrizes nacionais estabelecem que em todas as amostras essas células devem estar presentes, há a necessidade de implementar avaliações de monitoramento quanto à efetividade do método de rastreamento de lesões precursoras do câncer do colo uterino. O conhecimento científico adquirido pode permear a introdução de medidas diferenciadas para a prática.

\section{- CONCLUSÃO}

Neste estudo, 70\% dos exames citopatológicos foram realizados em mulheres entre 25 a 64 anos, população alvo das ações de promoção à saúde no âmbito da atenção primária. Se por um lado a idade, o contraceptivo oral, a TRH e o epitélio metaplásico atuaram como fatores facilitadores para a captura das células de ZT por outro lado, a fragilidade no rastreamento quanto à técnica da coleta pode demonstrar falhas na efetividade das ações e serviços na prática clínica. Isso pode ser verificado nesse estudo, onde quase um quarto da população estudada não teve a captura da ZT na realização do exame, demonstrando que a técnica de coleta pode e deve ser melhorada pelos profissionais que a realizam, bem como avaliada constantemente. A ausência dos dois epitélios nas amostras de coletas citopatológicas podem contribuir para a incidência de resultados falso-negativos e assim um retardo no diagnóstico precoce de lesões precursoras do câncer do colo uterino, corroborando com a hipótese inicial do estudo.

Nesse contexto, a enfermagem deve desenvolver um papel central no planejamento de ações que promovam o fortalecimento do processo de trabalho frente à necessidade do diagnóstico precoce desse tipo de câncer. Os achados desse estudo reforçam a necessidade de monitoramento constante da qualidade das coletas dos exames citopatológicos, a fim de fornecer uma assistência qualificada e 
resolutiva à população de mulheres assistidas.

Contudo, ressalta-se que os laudos analisados foram apenas aqueles ocorridos no âmbito do sistema público de saúde, o que significa uma visão parcial da realidade, configurando uma limitação do estudo. Sugere-se que as próximas pesquisas utilizem outros indicadores de qualidade e monitoramento das amostras de exames preventivos.

\section{O REFERÊNCIAS}

1. Clark CR, Soukup J, Riden H, Tovar D, Orton P, Burdick E, et al. Preventive care for low-income women in massachusetts post-health reform. Res J Womens Health. 2013; 23(6): 493-8.

2. Oliveira MV, Almeida MC. Prevalência de citologia inflamatória cervical em mulheres atendidas pelo laboratório de citologia da fundação de saúde de Vitória da Conquista: achados citológicos e agentes causais. C\&D-Revista Eletrônica da Fainor. [Internet] 2014; 7(1): 184-98 [acesso em 25 set 2015]. Disponível:

http://srv02.fainor.com.br/revista/index.php/memorias/article/view/278.

3. do Nascimento MI, e Silva GA, Monteiro GTR. História prévia de realização de teste de Papanicolaou e câncer do colo do útero: estudo caso-controle na Baixada Fluminense, Rio de Janeiro, Brasil. Cad Saúde Pública. 2012; 28(10): 1841-53.

4. Ministério da Saúde (BR). Secretaria de Atenção à Saúde. Departamento de Atenção Básica. Caderno de atenção básica - Controle dos cânceres do colo do útero e da mama. Brasília: Ministério da Saúde, 2013.

5. Thuler LCS, de Aguiar SS, Bergmann A. Determinantes do diagnóstico em estadio avançado do câncer do colo do útero no Brasil. Rev Bras Ginecol Obstet. 2014; 36(6): 237-43.

6. Ministério da Saúde (BR). Instituto Nacional do Câncer - Diretrizes brasileiras para o rastreamento do câncer do colo do útero. Brasília: Ministério da Saúde, 2011.

7. Nai GA, Souza KKG, Rodrigues ER, Barbosa RL. Presença de células da junção escamo-colunar em esfregaços cérvico-vaginais de mulheres acima de 40 anos. Rev Bras Ginecol Obstet. 2011; 33(3): 128-32.

8. da Silva DSM, Silva AMN, Brito LMO, Gomes SRL, Nascimento MDSB, Chein MBC. Rastreamento do câncer do colo do útero no Estado do Maranhão, Brasil. Ciênc. Saúde Colet. 2014; 19(4): 1163-70.

9. Bastos EA, Zardo LMG, Feitosa TMP, Almeida RT. Associação entre a qualidade da amostra e a detecção de atipias celulares no exame citopatológico do colo do Útero. Rev. Bras. Cancerol. 2012; 58(3): 445-52.

10. Ministério da Saúde (BR). Departamento de Informática do Sistema Único de Saúde [Internet]. População Residente em Santa Catarina; 2012 [acesso em 2 jul 2015]. Disponível:

http://tabnet.datasus.gov.br/cgi/deftohtm.exe?ibge/cnv/popsc.def

11. Albuquerque KM, Frias PG, Andrade CLT, Aquino EM, Menezes G, Szwarcwald CL. Cobertura do teste de papanicolaou e fatores associados à não-realização: um olhar sobre o Programa de Prevenção do Câncer do Colo do Útero em Pernambuco, Brasil. Cad Saúde Pública. 2009; 25(2): 301-9.

12. Cardoso CL, Santos EF, França AMB, Cavalcante TCS, Lima KBM. Análise da cobertura de exames citopatológicos no estado de Alagoas. Semina cienc. biol. saude. 2014; 2(2): 31-42.

13. World Health Organization (WHO). National cancer control programmes : policies and managerial guidelines. Geneva: World Health Organization; 2002.

14. Watson M, Saraiya M, Benard V, Coughlin SS, Flowers L, Cokkinides V, Schwenn M, Huang Y, Giuliano A. Burden of cervical cancer in the United States, 1998-2003. Cancer. 2008; 113(10): 2855-64.

15. Ministério da Saúde (BR). Instituto Nacional do Câncer - Programa Nacional de Controle do Câncer do Colo do Útero. Rio de Janeiro: Ministério da Saúde, 2011.

16. Iwamoto HH, Camargo FC, Miranda MP, Nunes JS, Barbosa IA. Mulheres que realizam Papanicolaou: contribuições para a estratégia saúde da família. Cogitare enferm. 2011; 16(3): 424-9. 
17. da Silva DW, de Andrade SM, Soares DA, Turini B, Schneck CA, Lopes MLS. Cobertura e fatores associados com a realização do exame Papanicolaou em Município do Sul do Brasil. Rev Bras. Ginecol. Obstet. 2006; 28(1): 24-31.

18. Rodrigues BC, Carneiro ACMO, da Silva TL, Solá ACN, Manzi NM, Schechtman NP, et al. Educação em Saúde para a Prevenção do Câncer Cérvico-uterino. Rev. Bras. Educ. Méd. 2012; 36(1): 149-54.

19. Baumgarten VZ, Longhi K, Bianchi MS, Gonçalves CV. Perfil sorológico das gestantes atendidas no pré-natal de um hospital universitário no sul do Brasil. Vittalle. 2011; 23(1): 67-74.

20. Fernandes RFM, Meincke SMK, Thumé E, Soares MC, Collet N, et al. Características do pré-natal de adolescentes em capitais das regiões sul e nordeste do Brasil. Texto Contexto Enferm. 2015; 24(1): 80-6.

21. Freitas HG, Thuler LCS. Monitoramento externo da qualidade dos exames citopatológicos cervicais realizados pelo Sistema Único de Saúde (SUS) no Estado de Mato Grosso do Sul. Rev Bras Ginecol Obstet. 2012; 34(8): 351-6.

22. Soares MBO, da Silva SR. Resultados de citologia oncótica em uma regional de saúde no período de 20072008. Rev. Rene. 2010; 11(n. esp): 23-31.

23. Moraes MN, Jerônimo CGF. Analysis of the results of cytopathological tests of uterine cervix. J Nurs Ufpe On Line. [Internet] 2015; 9(3): 7510-5 [acesso em 28 set 2015]. Disponível:

http://www.revista.ufpe.br/revistaenfermagem/index.php/revista/article/viewArticle/6561.

24. Sebold AC, Frigo J, Kohls M. Comparative evaluation of positive cytology, colposcopy and histopathology: a method of screening for cancer of the cervix. R. Pesq.: Cuid. Fundam Online. [Internet] 2012; 4(2): 2357-66 [acesso em 02 out 2015]. Disponível: http://www.seer.unirio.br/index.php/cuidadofundamental/article/view/1818.

25. Ferreira JEL, Alves MC, Martins MCV, Rosa MPRS, Gonçalves MC. Perfil da população atendida em um consultório de atendimento integral à saúde da mulher. Semina: Ciências Biológicas e da Saúde. 2015; 3(1): 12740.

26. da Silva MGP, de Almeida RT, Bastos EA, Nobre FF. Determinantes da detecção de atipias celulares no programa de rastreamento do câncer do colo do útero no Rio de Janeiro, Brasil. Rev Panam Salud Publica.2013; 34(2): 107-13.

27. Galvão EFB, da Silva MJM, Esteves FAM, Peres AL. Frequência de amostras insatisfatórias dos exames preventivos do câncer de colo uterino na rede pública de saúde, em município do agreste pernambucano. Rev Para Med. 2015; 29(2): 51-6.

28. Hwang LY, Ma Y, Shiboski SC, Farhat S, Jonte J, Moscicki AB. Active squamous metaplasia of the cervical epithelium is associated with subsequent acquisition of human papillomavirus 16 infection among healthy young women. J Infect Dis. 2012; 206(4): 504-11.

29. Santos ML, Moreno MS, Pereira VM. Exame de Papanicolaou: qualidade do esfregaço realizado por alunos de enfermagem. Rev. Bras. Cancerol. 2009; 55(1): 19-25. 